\title{
ON INAUDIBLE CURVATURE PROPERTIES OF CLOSED RIEMANNIAN MANIFOLDS
}

\author{
TERESA ARIAS-MARCO AND DOROTHEE SCHUETH
}

\begin{abstract}
Following Mark Kac, it is said that a geometric property of a compact Riemannian manifold can be heard if it can be determined from the eigenvalue spectrum of the associated Laplace operator on functions. On the other hand, D'Atri spaces, manifolds of type $\mathcal{A}$, probabilistic commutative spaces, $\mathfrak{C}$-spaces, $\mathfrak{T C}$-spaces, and $\mathfrak{G} \mathfrak{C}$-spaces have been studied by many authors as symmetric-like Riemannian manifolds. In this paper, we prove that for closed Riemannian manifolds, none of the properties just mentioned can be heard. Another class of interest is the class of weakly symmetric manifolds. We consider the local version of this property and show that weak local symmetry is another inaudible property of Riemannian manifolds.
\end{abstract}

\section{Introduction and Preliminaries}

If $M$ is a compact Riemannian without boundary one may consider the eigenvalue spectrum (with multiplicities) of the associated Laplace operator on functions and ask how much geometric information is encoded in the spectrum.

The classical heat invariants $a_{k}(M)\left(k \in \mathbb{N}_{0}\right)$ are coefficients of the asymptotic expansion of the heat kernel of $M$, and are thus spectrally determined in the above sense. In particular, the volume $a_{0}$ and the total scalar curvature $a_{1}$ of $M$ are spectrally determined. Note that each $a_{k}$ is some combination of expressions obtained from the Riemannian curvature tensor of $M$ by applying covariant derivatives and traces. Therefore, it is of particular interest to know which curvature properties of a Riemannian manifold are spectrally determined.

A geometric property is "inaudible", i.e., not determined by the spectrum, if there exist pairs of isospectral manifolds which differ with respect to this property. For example, it was shown in [11] that the maximum of the scalar curvature function on a Riemannian manifold is inaudible (in spite of $a_{1}$ ), and it was shown in [21] that the total squared norm of the Ricci tensor, $\int \mid$ ric $\left.\right|^{2}$, is inaudible, in spite of $a_{2}=\frac{1}{360} \int\left(5 \mathrm{scal}^{2}-2 \mid\right.$ ric $\left.\left.\right|^{2}+2|R|^{2}\right)$.

In the present paper we will prove inaudibility of several other curvature properties. For this we will use certain pairs of isospectral manifolds $N^{a+b, 0}$ and $N^{a, b}$ for $a, b>0$ which we will describe in Section 2. These manifolds are submanifolds of certain two-step Riemannian nilmanifolds and were introduced by Z.I. Szabó in [26]. Using them, Szabó proved, in particular, that local homogeneity is an inaudible property of Riemannian manifolds. More explicitly, he proved that $N^{a+b, 0}$ is a homogeneous manifold while $N^{a, b}$ is not even locally homogeneous.

The least restrictive of the curvature properties which we are going to consider is the so-called type $\mathcal{A}$ property. A Riemannian manifold $M$ is said to be of type $\mathcal{A}$ if its Ricci tensor ric is cyclic parallel; that is, if $\left(\nabla_{X}\right.$ ric $)(X, X)=0$ for all $X \in T M$. This class of Riemannian manifolds was

2000 Mathematics Subject Classification. 58J50, 58J53, 53C25, 53C30, 53C20, 22E25, 14J70.

Key words and phrases. Laplace operator, isospectral manifolds, curvature tensor, weak symmetry, D'Atri spaces, type $\mathcal{A}$ spaces, probabilistic commutative spaces, $\mathfrak{C}$-spaces, $\mathfrak{T} \mathfrak{C}$-spaces, $\mathfrak{G} \mathfrak{C}$-spaces.

The authors were partially supported by DFG Sonderforschungsbereich 647. The first author's work has also been supported by D.G.I. (Spain) and FEDER Project MTM 2007-65852 and the network MTM2008-01013-E/. 
introduced by A. Gray in [12] as one of the possible natural extensions of Einstein spaces. The type $\mathcal{A}$ property is clearly equivalent to $\operatorname{ric}(\dot{\gamma}(t), \dot{\gamma}(t))$ being constant in $t$ for each geodesic $\gamma$ in $M$. Manifolds of type $\mathcal{A}$ are known to be analytic [25] and to have constant sectional curvature [9]. In dimensions up to three, manifolds of type $\mathcal{A}$ have been classified [15] and are homogeneous. In dimensions $\geq 5$ there exist manifolds of type $\mathcal{A}$ which are not locally homogeneous [19]. In the four dimensional case it is still open whether the type $\mathcal{A}$ property implies local homogeneity. Four-dimensional homogeneous spaces of type $\mathcal{A}$ have recently been classified [1], [3].

Besides Einstein spaces, the most well-known examples of type $\mathcal{A}$ spaces are locally symmetric spaces (note that $\nabla R=0$ implies $\nabla$ ric $=0$ ), D'Atri spaces [9], and $\mathfrak{C}$-spaces [6].

D'Atri spaces were introduced by J.E. D'Atri and H.K. Nickerson in [9]. A Riemannian manifold is called a D'Atri space (cf. [27]) if the local geodesic symmetries (defined as $\sigma_{p}: \exp _{p}(X) \mapsto \exp _{p}(-X)$ on normal neighborhoods of $p$ ) preserve the Riemannian volume. Obviously, D'Atri spaces are a natural generalization of locally symmetric spaces (where the local geodesic symmetries are isometries). It is an open question whether each D'Atri space is locally homogeneous. The classification of D'Atri spaces has been obtained only in dimensions up to three [15]. In dimension four, partial classifications were obtained by J.T. Cho, K. Sekigawa and L. Vanhecke [22], [23], [8]; the classification of 4-dimensional homogeneous D'Atri spaces has recently been completed by the first author and O. Kowalski [1], [2], [3]. See [17] for references about D'Atri spaces and related topics. A useful characterization of D'Atri spaces was proved by J.E. D'Atri and H.K. Nickerson [9] and improved by Z.I. Szabó [25]; namely: A Riemannian manifold is a D'Atri space if, and only if, it satisfies the series of all odd Ledger conditions $L_{2 k+1}, k \geq 1$. The Ledger conditions $L_{m}, m \geq 2$, are an infinite series of curvature conditions derived from the so-called Ledger recurrence formula. This formula is derived using a calculation involving the Jacobi operator (see [17]). The explicit form of $L_{m}$ is known only for small values of $m$. The first nontrivial odd Ledger condition $L_{3}$ reads: $\left(\nabla_{X} \operatorname{ric}\right)(X, X)=0$ for all $X \in T M$; this is exactly the type $\mathcal{A}$ condition. Therefore, every D'Atri space is of type $\mathcal{A}$. The converse is not true [19].

$\mathfrak{C}$-spaces were introduced by J. Berndt and L. Vanhecke in [6]. By definition, a Riemannian manifold is a $\mathfrak{C}$-space if for each geodesic $\gamma$ in $M$ the eigenvalues of the associated field of Jacobi operators $R_{\dot{\gamma}(t)}:=R(., \dot{\gamma}(t)) \dot{\gamma}(t)$ are constant in $t$. For locally symmetric spaces this is always the case (by $\nabla R=0$ ), so $\mathfrak{C}$-spaces are another natural generalization of locally symmetric spaces. It is an open question whether every $\mathfrak{C}$-space is locally homogeneous. The classification of $\mathfrak{C}$-spaces has been obtained only in dimensions up to three [6]. Non-symmetric Damek-Ricci spaces were the first examples of D'Atri spaces which are not $\mathfrak{C}$-spaces [5]. However, it is an open question whether each $\mathfrak{C}$-space is a D'Atri space. Obviously, every $\mathfrak{C}$-space is of type $\mathcal{A}$ : If for each geodesic $\gamma$ in $M$ the eigenvalues of the operator $R_{\dot{\gamma}(t)}$ are constant in $t$ then so is its trace; but this trace is just $\operatorname{ric}(\dot{\gamma}(t), \dot{\gamma}(t))$. The converse is not true; recall that non-symmetric Damek-Ricci spaces are D'Atri spaces, hence of type $\mathcal{A}$, but are not $\mathfrak{C}$-spaces.

Weakly symmetric spaces were introduced by A. Selberg in [24]. A Riemannian manifold $M$ is called weakly symmetric if each $p \in M$ and each nontrivial geodesic $\gamma$ starting in $p$ there exists an isometry $f$ of $M$ which fixes $p$ and reverses $\gamma$ (equivalently: $d f_{p}(\dot{\gamma}(0))=-\dot{\gamma}(0)$ ). This is not Selberg's original definition, but was Z.I. Szabó's definition of what he called ray symmetry [25]. However, Selberg's and Szabó's definitions turned out to be equivalent [7]. Weak symmetry implies commutativity [24] which is defined as follows.

A commutative space is a homogeneous Riemannian manifold whose algebra of $I_{0}(M)$-invariant differential operators is commutative. Here, $I_{0}(M)$ denotes the connected component of the 
isometry group of $M$. J. Lauret gave the first example of a commutative space which is not weakly symmetric [18]. Every commutative space is a $\mathfrak{C}$-space [6].

Probabilistic commutative spaces were introduced by P.H. Roberts and H.D. Ursell in [20] for compact Riemannian manifolds from a probabilistic point of view. The general case has been treated by O. Kowalski and F. Prüfer in [14] and [16]. They characterized probabilistic commutative spaces as those Riemannian manifolds for which all Euclidean Laplacians $\tilde{\Delta}^{(k)}(k \in$ $\mathbb{N})$ commute. The Euclidean Laplacians are defined as follows: For $p \in M$ define a differential operator $\bar{\Delta}_{p}$ on a normal neighborhood of $p$ as the pullback of the (standard) Laplacian on the euclidean space $T_{p} M$ via the exponential map $\exp _{p}$. Then $\left(\tilde{\Delta}^{(k)} f\right)(p)$ is defined as $\left(\left(\bar{\Delta}_{p}\right)^{k} f\right)(p)$ for $f \in C^{\infty}(M)$. Since $\tilde{\Delta}^{(k)}$ is certainly invariant under isometries, the above characterization immediately implies that every commutative space is also probabilistic commutative. Moreover, every probabilistic commutative space is a D'Atri space [16]. Classifications of probabilistic commutative spaces are known only for dimension three [15]. For more information about probabilistic commutative spaces and Euclidean Laplacians we refer to [17].

Note that both the $\mathfrak{C}$ property and probabilistic commutativity (as well as the D'Atri and the type $\mathcal{A}$ property) are local properties of Riemannian manifolds. As mentioned above, both of them are implied by commutativity which, in turn, is implied by weak symmetry. Therefore, the $\mathfrak{C}$ property and probabilistic commutativity follow already in the case that some Riemannian covering of the manifold is weakly symmetric. In this context, let us introduce the following local version of weak symmetry:

Definition 1.1. A Riemannian manifold $M$ is called weakly locally symmetric if for every $p \in M$ there exists $\varepsilon>0$ such that for any unit speed geodesic $\gamma$ in $M$ with $\gamma(0)=p$ there exists an isometry of the distance ball $B_{\varepsilon}(p)$ which fixes $p$ and reverses $\left.\gamma\right|_{(-\varepsilon, \varepsilon)}$.

Local symmetry clearly implies weak local symmetry. The converse is not true: See [7] for examples of weakly symmetric spaces which are not locally symmetric. However, we note the following important fact which is analogous to the locally symmetric case:

Lemma 1.2. Let $M$ be a complete, simply connected, weakly locally symmetric Riemannian manifold. Then $M$ is weakly symmetric. In particular, the universal Riemannian covering of any complete, weakly locally symmetric Riemannian manifold is weakly symmetric.

Proof. Let $\gamma: \mathbb{R} \rightarrow M$ be a unit speed geodesic and $p=\gamma\left(t_{0}\right)$ any point on it. By weak local symmetry of $M$ there exists $\varepsilon>0$ and an isometry of $B_{\varepsilon}(p)$ which reverses $\left.\gamma\right|_{\left(t_{0}-\varepsilon, t_{0}+\varepsilon\right)}$. In particular, $s \mapsto \operatorname{ric}\left(\dot{\gamma}\left(t_{0}+s\right), \dot{\gamma}\left(t_{0}+s\right)\right)$ is an even function of $s \in(-\varepsilon, \varepsilon)$ and hence has derivative zero at $s=0$. So $t \mapsto \operatorname{ric}(\dot{\gamma}(t), \dot{\gamma}(t))$ has derivative zero at $t_{0}$. Since $t_{0}$ was arbitrary, this function is constant. This shows that $M$ is of type $\mathcal{A}$. As mentioned above, it follows that $M$ is analytic [25]. But on every complete, simply-connected, analytic Riemannian manifold, any isometry between open subsets of $M$ extends to an isometry of $M$ (see Corollary IV.6.4 in [13]). In particular, the isometries from the definition of weak local symmetry extend to isometries of $M$. Hence $M$ is weakly symmetric.

By the above discussion, Lemma 1.2 immediately implies that every complete, weakly locally symmetric Riemannian manifold is probabilistic commutative and a $\mathfrak{C}$-space. Lauret's aforementioned example [18] of a commutative (and hence probabilistic commutative and $\mathfrak{C}$-) space which is not weakly symmetric is in fact a complete, simply connected manifold; thus, by Lemma 1.2, it is not even weakly locally symmetric. 
In the following diagram we now summarize, in the setting of complete Riemannian manifolds, the relations between those classes of manifolds which we discussed above and which are defined by local properties:

$$
\begin{aligned}
& \subset \mathfrak{C} \text {-spaces } \\
\text { Loc. symm. } \subset \text { weakly loc. symm. } & \subset \text { prob. comm. } \subseteq \text { D'Atri }
\end{aligned}
$$

Here, $\subset$ means that the inclusion is strict while $\subseteq$ means that it is not known whether each D'Atri space is probabilistic commutative.

For our isospectral pairs of compact Riemannian manifolds $N^{a+b, 0}$ and $N^{a, b}$ (see Section 2) with $a, b>0$ we will prove in Section 3 that the first manifold is weakly locally symmetric, and we will prove in Section 4 that the second manifold is not even of type $\mathcal{A}$. Using (1) we can then conclude:

Main Theorem 1.3. Each of the following properties is an inaudible property of Riemannian manifolds: Weak local symmetry, the D'Atri property, probabilistic commutativity, the $\mathfrak{C}$ property, and the type $\mathcal{A}$ property.

Let us mention that there are two other (less studied) local properties of Riemannian manifolds which turn out to be inaudible by our examples, namely, the so-called $\mathfrak{T} \mathfrak{C}$ and $\mathfrak{G} \mathfrak{C}$ properties (see [4] or [5]). Each weakly locally symmetric space is a $\mathfrak{T C}$ space and a $\mathfrak{G} \mathfrak{C}$ space; on the other hand, each of these two properties implies the $\mathfrak{C}$ property. However, it is not known whether these properties are really stronger than the $\mathfrak{C}$ property and not equivalent to it.

Finally we remark here without proof that using the formula for the Ricci curvature from Lemma 4.3(i) below one can show that $\nabla$ ric $\neq 0$ for each of our manifolds $N^{a, b}$, including $N^{a, 0}$. In particular, none of our manifolds is locally symmetric (note that $\nabla R=0$ would imply $\nabla$ ric $=0$ ), or Einstein (which would imply $\nabla$ ric $=0$ ), or harmonic (which would imply Einstein). Whether any of these three properties is spectrally determined remains an open question.

\section{The MANifolds $N^{a, b}$}

Let $\mathfrak{v}$ and $\mathfrak{z}$ be euclidean vector spaces, each endowed with a fixed inner product, and let $\mathcal{L}$ be a cocompact lattice in $\mathfrak{z}$.

Definition 2.1. Given the above data, one associates with any linear map $j: \mathfrak{z} \rightarrow \mathfrak{s o}(\mathfrak{v})$ the following:

(i) The two-step nilpotent metric Lie algebra $\mathfrak{g}(j)$ with underlying vector space $\mathfrak{v} \oplus \mathfrak{z}$, whose inner product is given by letting $\mathfrak{v}$ and $\mathfrak{z}$ be orthogonal and taking the given inner product on each factor, and whose Lie bracket $[,]^{j}$ is defined by letting $\mathfrak{z}$ be central, $[\mathfrak{v}, \mathfrak{v}]^{j} \subseteq \mathfrak{z}$ and $\left\langle j_{Z} X, Y\right\rangle=\left\langle Z,[X, Y]^{j}\right\rangle$ for all $X, Y \in \mathfrak{v}$ and $Z \in \mathfrak{z}$.

(ii) The two-step simply connected nilpotent Lie group $G(j)$ whose Lie algebra is $\mathfrak{g}(j)$, and the left invariant Riemannian metric $g(j)$ on $G(j)$ which coincides with the chosen inner product on $\mathfrak{g}(j)=T_{e} G(j)$. Note that the Lie group exponential map $\exp ^{j}: \mathfrak{g}(j) \rightarrow G(j)$ is a diffeomorphism because $G(j)$ is simply connected and nilpotent. Moreover, by the Campbell-Baker-Hausdorff formula, $\exp ^{j}(X, Z) \cdot \exp ^{j}(Y, W)=\exp ^{j}(X+Y, Z+W+$ $\left.\frac{1}{2}[X, Y]^{j}\right)$ for all $X, Y \in \mathfrak{v}$ and $Z, W \in \mathfrak{z}$.

(iii) The two-step nilpotent Lie group $G(j) / \exp ^{j}(\mathcal{L})$. Note that $\exp ^{j}(\mathcal{L})$ is a discrete central subgroup of $G(j)$, and that $g(j)$ induces a left invariant metric, which we denote again $g(j)$, on $G(j) / \exp ^{j}(\mathcal{L})$. 
(iv) The submanifolds $\tilde{N}(j):=\left\{\exp ^{j}(X, Z)|X \in \mathfrak{v}| X \mid,=1, Z \in \mathfrak{z}\right\}$ of $G(j)$ and $N(j):=$ $\tilde{N}(j) / \exp ^{j}(\mathcal{L})$ of $G(j) / \exp ^{j}(\mathcal{L})$, each endowed with the Riemannian metric induced by $g(j)$. Note that $\tilde{N}(j)$ is indeed invariant under multiplication by elements of $\exp ^{j} \mathfrak{z}$ because of $\exp ^{j}(X, Z) \cdot \exp ^{j}(0, W)=\exp ^{j}(X, Z+W)$. Moreover, $\tilde{N}(j)$ is diffeomorphic to $S^{\text {dimv-1}} \times \mathfrak{z}$, and that $N(j)$ is compact and diffeomorphic to $S^{\text {dimv-1 }} \times(\mathfrak{z} / \mathcal{L})$, the product of a sphere and a torus. The canonical projection $\tilde{N}(j) \rightarrow N(j)$ is a Riemannian covering.

Definition 2.2. Two linear maps $j, j^{\prime}: \mathfrak{z} \rightarrow \mathfrak{s o}(\mathfrak{v})$ are called isospectral if for each $Z \in \mathfrak{z}$, the maps $j_{Z}, j_{Z}^{\prime} \in \mathfrak{s o}(\mathfrak{v})$ have the same eigenvalues (with multiplicities) in $\mathbb{C}$.

Proposition 2.3 (see [11]). Let $j, j^{\prime}: \mathfrak{z} \rightarrow \mathfrak{s o}(\mathfrak{v})$ be isospectral, and let $\mathcal{L}$ be a cocompact lattice in $\mathfrak{z}$. Then the associated closed Riemannian manifolds $N(j)$ and $N\left(j^{\prime}\right)$, obtained as above, are isospectral for the Laplace operator on functions.

Remark 2.4. If $j, j^{\prime}: \mathfrak{z} \rightarrow \mathfrak{s o}(\mathfrak{v})$ are both of Heisenberg type, that is, if $j_{Z}^{2}=j_{Z}^{\prime 2}=-|Z|^{2} \mathrm{Id}_{\mathfrak{v}}$ for all $Z \in \mathfrak{z}$, then $j$ and $j^{\prime}$ are obviously isospectral because the eigenvalues for both of them are $\pm i|Z|$, each with multiplicity $(\operatorname{dim} \mathfrak{v}) / 2$.

Definition 2.5. Let $\mathbb{H}=\operatorname{span}\{1, i, j, k\}$ denote the algebra of quaternions with the usual multiplication, endowed with the inner product for which $\{1, i, j, k\}$ is an orthonormal basis. For $a, b \in \mathbb{N}_{0}$ with $a+b>0$ define $\mathfrak{v}$ as the direct orthogonal sum of $a+b$ copies of $\mathbb{H}$, let $\mathfrak{z}:=\operatorname{span}\{i, j, k\}$ be the space of pure quaternions, and let $\mathcal{L}:=\operatorname{span}_{\mathbb{Z}}\{i, j, k\}$ be the standard lattice in $\mathfrak{z}$. Define $j^{a, b}: \mathfrak{z} \rightarrow \mathfrak{s o}(\mathfrak{v})$ by

$$
j_{Z}^{a, b}: \mathfrak{v} \ni\left(X_{1}, \ldots, X_{a}, Y_{1}, \ldots, Y_{b}\right) \mapsto\left(X_{1} Z, \ldots, X_{a} Z, Z Y_{1}, \ldots, Z Y_{b}\right) \in \mathfrak{v} .
$$

We denote the resulting Riemannian manifolds by

$$
N^{a, b}:=N\left(j^{a, b}\right), \operatorname{resp} . \tilde{N}^{a, b}:=\tilde{N}\left(j^{a, b}\right) .
$$

\section{Remark 2.6.}

(i) For all pairs $(a, b) \in \mathbb{N}_{0}^{2}$ with fixed sum $a+b=(\operatorname{dim} \mathfrak{v}) / 4>0$ the associated Riemannian manifolds $N^{a, b}$ are obviously of Heisenberg type and thus mutually isospectral by Proposition 2.3 and Remark 2.4. Independently of Proposition 2.3 from [11] this had previously been shown by Z.I. Szabó in [26] using explicit calculations.

(ii) Of particular interest to us will be the isospectrality of the pairs $N^{a+b, 0}$ and $N^{a, b}$ for $a, b>0$ : We will show in this paper that $N^{a+b, 0}$ is weakly locally symmetric, and thus, in particular, a D'Atri space, while $N^{a, b}$ for $a, b>0$ is not a D'Atri space and not even of type $\mathcal{A}$. We will also exhibit several other geometric properties that these isospectral pairs do not share. The simplest such example will be given by pair of isospectral ten-dimensional closed Riemannian manifolds $N^{2,0}$ and $N^{1,1}$.

Proposition 2.7. For every $a \in \mathbb{N}$ the manifolds $N^{a, 0}$ and $\tilde{N}^{a, 0}$ are homogeneous.

Proof. Although this was already shown in [26], we include an explicit proof here for convenience of the reader. In the general context of Definition 2.1, note that if $A \in \mathrm{O}(\mathfrak{v})$ and $C \in \mathrm{O}(\mathfrak{z})$ are two orthogonal maps which satisfy

$$
A j_{Z} A^{-1}=j_{C Z} \text { for all } Z \in \mathfrak{z}
$$

then $[A X, A Y]=C[X, Y]$ for all $X, Y \in \mathfrak{v}$; hence

$$
(A, C): \mathfrak{v} \oplus \mathfrak{z} \ni(X, Z) \mapsto(A X, C Z) \in \mathfrak{v} \oplus \mathfrak{z}
$$


is an isometric Lie algebra isomorphism of $\mathfrak{g}(j)$ and is, thus, the differential of an isometric Lie group automorphism

$$
f_{A, C}:(G(j), g(j)) \ni \exp ^{j}(X, Z) \mapsto \exp ^{j}(A X, C Z) \in(G(j), g(j)),
$$

where $X$ and $Z$ denote elements of $\mathfrak{v}$ and $\mathfrak{z}$, respectively. Note that $f_{A, C}$ restricts to an isometry of $\tilde{N}(j)$. Now for $j:=j^{a, 0}$ and $\mathfrak{v}=\mathbb{H}^{a}$, the pair $(A, \mathrm{Id})$ satisfies (2) for any

$$
A \in \operatorname{Sp}(a):=\left\{A \in \mathrm{O}\left(\mathbb{H}^{a}\right) \mid A(X \lambda)=A(X) \lambda \text { for all } \lambda \in \mathbb{H}, X \in \mathbb{H}^{a}\right\} .
$$

Write $\exp :=\exp ^{j}$. Given an arbitrary pair of points in $\tilde{N}^{a, 0}$, say, $\exp (X, Z), \exp (Y, W)$ with $X, Y \in \mathfrak{v}, Z, W \in \mathfrak{z}$, we choose $A \in \operatorname{Sp}(a)$ such that $A X=Y$. This is possible because $\operatorname{Sp}(a)$ acts transitively on the unit sphere in $\mathbb{H}^{a}$. Then the isometry $f_{A, \text { Id }} \operatorname{maps} \exp (X, Z)$ to $\exp (Y, Z)$. Finally, left translation by $\exp (0, W-Z)$ is another isometry of $G^{a, 0}$ which restricts to $\tilde{N}^{a, 0}$, and it maps $\exp (Y, Z)$ to $\exp (Y, W)$. Therefore $\tilde{N}^{a, 0}$ is homogeneous; since the isometries we used commute with the left action of $\exp (\mathcal{L})$ and thus descend to isometries of $N^{a, 0}$, the latter is homogeneous, too.

Remark 2.8. Z.I. Szabó also proved in [26] that $N^{a, b}$ is not locally homogeneous if both $a$ and $b$ are nonzero. More precisely, he showed that in this case any isometry of the universal covering $\tilde{N}^{a, b}$ leaves $\exp \left(\mathfrak{v}_{1} \times \mathfrak{z}\right)$ and $\exp \left(\mathfrak{v}_{2} \times \mathfrak{z}\right)$ invariant, where $\mathfrak{v}_{1}:=\mathbb{H}^{a} \times\{0\}, \mathfrak{v}_{2}:=\{0\} \times \mathbb{H}^{b} \subset$ $\mathbb{H}^{a+b}=\mathfrak{v}$. Szabó's isospectral pairs $N^{a+b, 0}$ and $N^{a, b}$ thus exhibited the surprising fact that local homogeneity is an inaudible property of Riemannian manifolds.

\section{WEAK LOCAL SYMMETRY OF $N^{a, 0}$}

In this section we are going to show that the Riemannian manifolds $\tilde{N}^{a, 0}$ are weakly symmetric (Theorem 3.1); in particular, since $\tilde{N}^{a, 0}$ and $N^{a, 0}$ are locally isometric, the manifold $N^{a, 0}$ is weakly locally symmetric.

Theorem 3.1. For any $a \in \mathbb{N}$ the Riemannian manifold $\tilde{N}^{a, 0}$ is weakly symmetric. In particular, $N^{a, 0}$ is weakly locally symmetric.

As a preparation for the proof we first state:

Lemma 3.2 (cp. [11]). In the general context of Definition 2.1, for any $p:=\exp ^{j}(x, z) \in \tilde{N}(j)$ with $x \in \mathfrak{v},|x|=1, z \in \mathfrak{z}$ we have

$$
T_{p} \tilde{N}(j)=L_{p *}\{(X, Z) \mid X \in \mathfrak{v}, X \perp x, Z \in \mathfrak{z}\} .
$$

In particular, $L_{p *}(x, 0)$ is a unit normal vector to $T_{p} \tilde{N}(j)$ in $T_{p} G(j)$.

Proof. We write exp $:=\exp ^{j}$. The definition of $\tilde{N}(j)$ implies $T_{p} \tilde{N}(j)=\exp _{*(x, z)}\left(x^{\perp} \times \mathfrak{z}\right)$, where $x^{\perp}$ is the orthogonal complement of $\operatorname{span}\{x\}$ in $\mathfrak{v}$. Note that

$$
\exp _{*(x, z)}=L_{p *}\left(\operatorname{Id}-\frac{1}{2} \operatorname{ad}_{x}\right) ;
$$

this identity holds in any two-step nilpotent Lie group and follows from the Campbell-BakerHausdorff formula. In particular, $\exp _{*(x, z)}$ and $L_{p *}$ coincide on $\mathfrak{z}$, hence $L_{p * \mathfrak{z}} \subset T_{p} \tilde{N}(j)$. Finally, for any $Y \in x^{\perp}$, the element $\exp _{*(x, z)} Y=L_{p *}\left(Y-\frac{1}{2}[x, Y]\right)$ of $T_{p} \tilde{N}(j)$ differs from $L_{p *} Y$ by an element of $L_{p * \mathfrak{z}}$. Consequently, $L_{p *} Y$ lies in $T_{p} \tilde{N}(j)$. 
Proof of Theorem 3.1. Again we use the abbreviations $j:=j^{a, 0}$ and $\exp :=\exp ^{j}$. We write vectors in $\mathfrak{v}=\mathbb{H}^{a}$ in the form $X=\left(X_{1}, \ldots, X_{a}\right)$ with $X_{1}, \ldots, X_{a} \in \mathbb{H}$.

In order to show that $\tilde{N}^{a, 0}$ is weakly symmetric, we must, for any given point $p \in \tilde{N}^{a, 0}$ and any given tangent vector at $p$, find an isometry of $\tilde{N}^{a, 0}$ which fixes $p$ and whose differential maps the given tangent vector to its negative.

Since $\tilde{N}^{a, 0}$ is homogeneous it suffices to consider the case $p:=\exp ((1,0, \ldots, 0), 0)$. We then have, by Lemma 3.2,

$$
T_{p} \tilde{N}^{a, 0}=L_{p *}\left\{\left(\left(X_{1}, \ldots, X_{a}\right), Z\right) \mid X_{1}, Z \in \mathfrak{z}=\operatorname{span}\{i, j, k\}, X_{2}, \ldots, X_{a} \in \mathbb{H}\right\} .
$$

Let $L_{p *}(X, Z)$ be any tangent vector in $T_{p} \tilde{N}^{a, 0}$ and write $X=\left(X_{1}, X^{\prime}\right)$ with $X^{\prime}=\left(X_{2}, \ldots, X_{a}\right)$. Choose $W \in \operatorname{span}\{i, j, k\}$ such that $W \perp \operatorname{span}\left\{X_{1}, Z\right\}$ and $|W|=1$. Define $A_{1} \in \mathrm{O}(\mathfrak{v})$ by $A_{1} Y:=-W Y W$, and define $C \in \mathrm{O}(\mathfrak{z})$ by $C Z:=-W Z W$. Note that $A_{1}$ and $C$ act on $\mathfrak{v}$ and $\mathfrak{z}$, respectively, as $\operatorname{Id}_{\text {span }\{1, W\}}-\operatorname{Id}_{\text {span }\{1, W\}^{\perp}}$. The pair $\left(A_{1}, C\right)$ then satisfies (2). In fact, for all $U \in \mathfrak{z}$ and all $Y \in \mathfrak{v}:$

$$
\begin{aligned}
A_{1} j_{U} A_{1}^{-1}(Y) & =A_{1} j_{U}(-W Y W)=A_{1}(-W Y W U)=W W Y W U W=-Y W U W \\
& =Y \cdot C U=j_{C U} Y .
\end{aligned}
$$

Define the associated isometry $f_{1}:=f_{A_{1}, C}$ as in the proof of Proposition 2.7. Then $f_{1}$ fixes $p$ because of $A_{1}(1,0)=(-W W, 0)=(1,0)$. Moreover, the differential of $f_{1}$ at $e$ is $\left(A_{1}, C\right) \in$ $\mathrm{O}(\mathfrak{v} \oplus \mathfrak{z})$ and $f_{1}$ is a Lie group automorphism of $G^{a, 0}$, so its differential at $p$ maps $L_{p *}\left(\left(X_{1}, X^{\prime}\right), Z\right)$ to $L_{p *}\left(A_{1} X, C Z\right)$. This is equal to $L_{p *}\left(\left(-X_{1},-W X^{\prime} W\right),-Z\right)$ because of $X_{1}, Z \in \operatorname{span}\{1, W\}^{\perp}$.

For $\ell=2, \ldots, a$ now choose $c_{\ell} \in \mathbb{H}$ with $\left|c_{\ell}\right|=1$ such that $c_{\ell} W X_{\ell} W=X_{\ell}$. (Note that $c_{\ell}$ is unique if $X_{\ell} \neq 0$ and arbitrary else.) Define $A_{2} \in \operatorname{Sp}(a) \subset \mathrm{O}(\mathfrak{v})$ by $A_{2}\left(Y_{1}, \ldots, Y_{a}\right):=$ $\left(Y_{1}, c_{2} Y_{2}, \ldots, c_{a} Y_{a}\right)$. Then $\left(A_{2}, \mathrm{Id}\right)$ satisfies $(2)$, the corresponding isometry $f_{2}:=f_{A_{2}, \mathrm{Id}}$ fixes $p$, and its differential at $p$ maps $L_{p *}\left(\left(-X_{1},-W X^{\prime} W\right),-Z\right)$ to $L_{p *}\left(\left(-X_{1},-X^{\prime}\right),-Z\right)$. The isometry $f:=f_{2} \circ f_{1}$ of $\tilde{N}^{a, 0}$ hence fixes $p$ and maps our given tangent vector $L_{p *}\left(\left(X_{1}, X^{\prime}\right), Z\right)$ to its negative, as wished.

Note that the isometry $f_{1}$ in the proof of Theorem 3.1 will in general not descend to the quotient manifold $N^{a, 0}$ because the condition $C \mathcal{L} \subset \mathcal{L}$ will not hold in general. So we cannot conclude weak symmetry of $N^{a, 0}$ but only weak local symmetry.

\section{Failure of the type $\mathcal{A}$ COndition for $N^{a, b}$ with $a, b>0$}

In this section we will show that for $a, b>0$ the Riemannian manifolds $N^{a, b}$ are not of type $\mathcal{A}$ (Theorem 4.4). We first introduce some notation and compute some formulas for the Ricci curvature of the manifolds $\tilde{N}(j)$ from Definition 2.1 in the case that $j$ is of Heisenberg type (Lemma 4.3). In particular, these formulas will hold for our manifolds $\tilde{N}^{a, b}$.

\section{Notation 4.1.}

(i) Recall the notation from Definition 2.1. In the following, let $j: \mathfrak{z} \rightarrow \mathfrak{s o}(\mathfrak{v})$ be any linear map (not necessarily one of our maps $\left.j^{a, b}\right)$. We write $[]:,=[,]^{j}, \mathfrak{g}:=\mathfrak{g}(j), \exp :=\exp ^{j}$, $G:=G(j), g:=g(j)$. Inner products $\langle$,$\rangle and norms |$.$| will refer to g$ (either on $\tilde{N}$ or on $G)$. We denote the Levi Civita connection of $\tilde{N}$ by $\nabla$ and that of $(G, g)$ by $\nabla^{G}$. The Ricci tensor of $\tilde{N}$ will be denoted by ric and that of $G$ by ric ${ }^{G}$.

(ii) For any $p=\exp (x, z) \in \tilde{N}$, where $x \in \mathfrak{v},|x|=1, z \in \mathfrak{z}$, we let

$$
\nu_{p}:=L_{p *}(x, 0) \in T_{p} G \text {. }
$$


Recall from Lemma 3.2 that $\nu_{p}$ is a unit normal vector to $T_{p} \tilde{N}$.

(iii) We denote by $S$ the Weingarten map associated with the unit normal field $\nu$ on the submanifold $\tilde{N}$ of $(G, g)$. More precisely, $S$ is the endomorphism field on $\tilde{N}$ given by

$$
S_{p}(Y)=-\nabla_{Y}^{G} \nu \in T_{p} \tilde{N}
$$

for all $p \in \tilde{N}, Y \in T_{p} \tilde{N}$.

(iv) We will often identify vectors in $T_{p} G=L_{p *} \mathfrak{g}$ with their preimage in $\mathfrak{g}$. That is, we regard $Y \in T_{p} G$ as the value in $p$ of the corresponding left invariant vector field $Y$ on $G$. Correspondingly, we will decompose $Y \in T_{p} G$ as

$$
Y=Y^{\mathfrak{v}}+Y^{\mathfrak{z}}
$$

with $Y^{\mathfrak{v}} \in \mathfrak{v}, Y^{\mathfrak{z}} \in \mathfrak{z}$.

Lemma 4.2. Let $p=\exp (x, z) \in \tilde{N}$, where $x \in \mathfrak{v},|x|=1, z \in \mathfrak{z}$. Then

$$
S_{p}(Y)=-Y^{\mathfrak{v}}-\frac{1}{2}\left[Y^{\mathfrak{v}}, x\right]+\frac{1}{2} j_{Y^{\mathfrak{s}}} x .
$$

Proof. From the definition of $\nu$ it follows that $\nabla_{Y}^{G} \nu=Y^{\mathfrak{v}}+\nabla_{Y}^{G} x$, where $x$ now denotes the left invariant vector field associated to $x \in \mathfrak{v}$. By general formulas for left invariant vector fields on two-step nilpotent Lie groups (see, e.g., [10]), one has $\nabla_{Y}^{G} x=\frac{1}{2}\left[Y^{\mathfrak{v}}, x\right]-\frac{1}{2} j_{Y^{\mathfrak{z}}} x$.

Lemma 4.3. Assume that $j$ is of Heisenberg type. Let $p=\exp (x, z) \in \tilde{N}$, where $x \in \mathfrak{v},|x|=1$, $z \in \mathfrak{z}$. Then for all $Y_{1}, Y_{2}, Y \in T_{p} \tilde{N}$ we have

$$
\begin{aligned}
\operatorname{ric}_{p}\left(Y_{1}, Y_{2}\right)= & \left(\operatorname{dim} \mathfrak{v}-2-\frac{1}{2} \operatorname{dim} \mathfrak{z}\right)\left\langle Y_{1}^{\mathfrak{v}}, Y_{2}^{\mathfrak{v}}\right\rangle+\left(\frac{1}{4} \operatorname{dim} \mathfrak{v}-\frac{1}{2}\right)\left\langle Y_{1}^{\mathfrak{z}}, Y_{2}^{\mathfrak{z}}\right\rangle \\
& +\frac{1}{2}\left\langle\left[Y_{1}^{\mathfrak{v}}, x\right],\left[Y_{2}^{\mathfrak{v}}, x\right]\right\rangle+\frac{1}{2}(\operatorname{dim} \mathfrak{v}-2)\left\langle j_{Y_{1}^{\mathfrak{3}}} Y_{2}^{\mathfrak{v}}+j_{Y_{2}^{\mathfrak{z}}} Y_{1}^{\mathfrak{v}}, x\right\rangle,
\end{aligned}
$$

$$
\left(\nabla_{Y} \operatorname{ric}\right)(Y, Y)=\left\langle\left[Y^{\mathfrak{v}}, x\right],\left[j_{Y^{\mathfrak{\jmath}}} Y^{\mathfrak{v}}, x\right]\right\rangle \text {. }
$$

Proof. The Ricci curvature ric of $\tilde{N}$ satisfies the following formula which holds in the general context of submanifolds of codimension one and can easily be derived from the Gauss equation:

$$
\operatorname{ric}_{p}(Y, Y)=\operatorname{ric}_{p}^{G}(Y, Y)-K^{G}\left(\operatorname{span}\left\{Y, \nu_{p}\right\}\right)|Y|^{2}+\operatorname{trace}\left(S_{p}\right)\left\langle S_{p}(Y), Y\right\rangle-\left|S_{p}(Y)\right|^{2}
$$

for all $p \in \tilde{N}$ and $Y \in T_{p} \tilde{N}$, where $K^{G}$ denotes sectional curvature in $(G, g)$. Now let $p=$ $\exp (x, z) \in \tilde{N}$ and $Y=Y^{\mathfrak{v}}+Y^{\mathfrak{z}} \in T_{p} \tilde{N} \subset T_{p} G$. Since $(G, g)$ is of Heisenberg type, we have $($ see $[5])$ :

$$
\operatorname{ric}_{p}^{G}(Y, Y)=-\frac{1}{2} \operatorname{dim} \mathfrak{z} \cdot\left|Y^{\mathfrak{v}}\right|^{2}+\frac{1}{4} \operatorname{dim} \mathfrak{v} \cdot\left|Y^{\mathfrak{z}}\right|^{2} .
$$

Moreover, by a general formula for left invariant metrics we have, noting that $\mathrm{ad}^{2}=0$ and $\nabla_{x}^{G} x=\frac{1}{2}[x, x]=0$ :

$$
\begin{aligned}
K^{G}\left(\operatorname{span}\left\{Y, \nu_{p}\right\}\right)|Y|^{2} & =\left|\nabla_{Y}^{G} x\right|^{2}-|[Y, x]|^{2}-\left\langle\nabla_{Y}^{G} Y, \nabla_{x}^{G} x\right\rangle-\left\langle\operatorname{ad}_{x}^{2} Y, Y\right\rangle \\
& =\frac{1}{4}\left|\left[Y^{\mathfrak{v}}, x\right]\right|^{2}+\frac{1}{4}\left|j_{Y^{\mathfrak{s}}} x\right|^{2}-\left|\left[Y^{\mathfrak{v}}, x\right]\right|^{2}-0-0=-\frac{3}{4}\left|\left[Y^{\mathfrak{v}}, x\right]\right|^{2}+\frac{1}{4}\left|Y^{\mathfrak{z}}\right|^{2},
\end{aligned}
$$

where for the last term we have used the Heisenberg type property and $|x|=1$. Moreover, by Lemma 4.2 , we have $\operatorname{trace}\left(S_{p}\right)=-(\operatorname{dim} \mathfrak{v}-1),\left\langle S_{p}(Y), Y\right\rangle=-\left|Y^{\mathfrak{v}}\right|^{2}-\left\langle j_{Y^{\mathfrak{\jmath}}} Y^{\mathfrak{v}}, x\right\rangle$, and 
$\left|S_{p}(Y)\right|^{2}=\left|Y^{\mathfrak{v}}\right|^{2}+\frac{1}{4}\left|\left[Y^{\mathfrak{v}}, x\right]\right|^{2}+\frac{1}{4}\left|Y^{\mathfrak{b}}\right|^{2}+\left\langle j_{Y^{\mathfrak{3}}} Y^{\mathfrak{v}}, x\right\rangle$. In our setting, formula (4) thus becomes

$$
\begin{aligned}
\operatorname{ric}_{p}(Y, Y)= & -\frac{1}{2} \operatorname{dim} \mathfrak{z} \cdot\left|Y^{\mathfrak{v}}\right|^{2}+\frac{1}{4} \operatorname{dim} \mathfrak{v} \cdot\left|Y^{\mathfrak{b}}\right|^{2}+\frac{3}{4}\left|\left[Y^{\mathfrak{v}}, x\right]\right|^{2}-\frac{1}{4}\left|Y^{\mathfrak{z}}\right|^{2} \\
& +(\operatorname{dim} \mathfrak{v}-1)\left|Y^{\mathfrak{v}}\right|^{2}+(\operatorname{dim} \mathfrak{v}-1)\left\langle j_{Y^{\mathfrak{z}}} Y^{\mathfrak{v}}, x\right\rangle \\
& -\left|Y^{\mathfrak{v}}\right|^{2}-\frac{1}{4}\left|\left[Y^{\mathfrak{v}}, x\right]\right|^{2}-\frac{1}{4}\left|Y^{\mathfrak{z}}\right|^{2}-\left\langle j_{Y^{\mathfrak{z}}} Y^{\mathfrak{v}}, x\right\rangle \\
= & \left(\operatorname{dim} \mathfrak{v}-2-\frac{1}{2} \operatorname{dim} \mathfrak{z}\right)\left|Y^{\mathfrak{v}}\right|^{2}+\left(\frac{1}{4} \operatorname{dim} \mathfrak{v}-\frac{1}{2}\right)\left|Y^{\mathfrak{z}}\right|^{2} \\
& +\frac{1}{2}\left|\left[Y^{\mathfrak{v}}, x\right]\right|^{2}+(\operatorname{dim} \mathfrak{v}-2)\left\langle j_{Y^{\mathfrak{z}}} Y^{\mathfrak{v}}, x\right\rangle .
\end{aligned}
$$

Statement (i) now follows by polarization. In order to prove (ii) we may assume $\left|Y^{\mathfrak{v}}\right|=1$ by rescaling $Y$. Then the curve $c: \mathbb{R} \rightarrow G$ defined by

$$
c(t):=\exp \left(\cos t \cdot x+\sin t \cdot Y^{\mathfrak{v}}, z+t Y^{\mathfrak{z}}+\frac{1}{2} t\left[x, Y^{\mathfrak{v}}\right]\right)
$$

is a curve in $\tilde{N}$, and $c(0)=p$. By $(3)$,

$$
\dot{c}(t)=\exp _{* c(t)}\left(-\sin t \cdot x+\cos t \cdot Y^{\mathfrak{v}}+Y^{\mathfrak{z}}+\frac{1}{2}\left[x, Y^{\mathfrak{v}}\right]\right)=L_{c(t) *} Y(t),
$$

where $Y(t):=-\sin t \cdot x+\cos t \cdot Y^{\mathfrak{v}}+Y^{\mathfrak{z}}$.

In particular, $\dot{c}(0)=Y$. We now have, extending $Y \in T_{p} \tilde{N}$ as the vector field $\dot{c}$ along $c$ :

$$
\left(\nabla_{Y} \text { ric }\right)(Y, Y)=\left.\frac{d}{d t}\right|_{t=0} \operatorname{ric}_{c(t)}(\dot{c}(t), \dot{c}(t))-2 \operatorname{ric}_{p}\left(Y, \nabla_{Y} \dot{c}\right) .
$$

We observe that $\left|Y^{\mathfrak{v}}(t)\right|^{2} \equiv 1,\left|Y^{\mathfrak{z}}(t)\right|^{2} \equiv\left|Y^{\mathfrak{z}}\right|^{2},\left|\left[Y^{\mathfrak{v}}(t), \cos t \cdot x+\sin t \cdot Y^{\mathfrak{v}}\right]\right|^{2} \equiv\left|\left[Y^{\mathfrak{v}}, x\right]\right|^{2}$, and $\left\langle j_{Y^{\mathfrak{z}}}\left(-\sin t \cdot x+\cos t \cdot Y^{\mathfrak{v}}\right), \cos t \cdot x+\sin t \cdot Y^{\mathfrak{v}}\right\rangle \equiv\left\langle Y^{\mathfrak{z}},\left[Y^{\mathfrak{v}}, x\right]\right\rangle$ for all $t$. The first term on the right hand side of (5) therefore vanishes by (i). Concerning the second term, note that

$$
\nabla_{Y}^{G} \dot{c}=\dot{Y}(0)+\nabla_{Y}^{G} Y=-x-j_{Y^{\mathfrak{3}}} Y^{\mathfrak{v}} .
$$

By Lemma 3.2, orthogonal projection of this vector to $T_{p} \tilde{N}$ gives

$$
\nabla_{Y} \dot{c}=-j_{Y^{\mathfrak{z}}} Y^{\mathfrak{v}}+\left\langle j_{Y^{\mathfrak{s}}} Y^{\mathfrak{v}}, x\right\rangle x .
$$

In particular, $\nabla_{Y} \dot{c}$ lies in $L_{p *} \mathfrak{v}$ and is perpendicular to $Y^{\mathfrak{v}}$. When we compute $\operatorname{ric}_{p}\left(Y, \nabla_{Y} \dot{c}\right)$ using the formula from (i), the first two terms on the right hand side thus vanish. The fourth term vanishes, too, because the vector $j_{Y^{\mathfrak{z}}}\left(-j_{Y^{\mathfrak{z}}} Y^{\mathfrak{v}}+\left\langle j_{Y^{\mathfrak{z}}} Y^{\mathfrak{v}}, x\right\rangle x\right)+j_{0} Y^{\mathfrak{v}}=\left|Y^{\mathfrak{b}}\right|^{2} Y^{\mathfrak{v}}+\langle\ldots\rangle j_{Y^{\mathfrak{z}}} x$ is perpendicular to $x$. Hence we remain with the third term only and obtain $\operatorname{ric}_{p}\left(Y, \nabla_{Y} \dot{c}\right)=$ $\frac{1}{2}\left\langle\left[Y^{\mathfrak{v}}, x\right],\left[-j_{Y_{\mathfrak{\jmath}}^{\mathfrak{j}}} Y^{\mathfrak{v}}, x\right]\right\rangle$. Statement (ii) now follows from (5).

Theorem 4.4. For $a, b>0$ the Riemannian manifolds $N^{a, b}$ and $\tilde{N}^{a, b}$ are not of Type $\mathcal{A}$.

Proof. Since the type $\mathcal{A}$ condition is a local condition and since $N^{a, b}$ and $\tilde{N}^{a, b}$ are locally isometric, it suffices to prove the statement for $\tilde{N}^{a, b}$. We write $j:=j^{a, b},[]:,=[,]^{j}$, exp $:=\exp ^{j}$ and continue to use the notation of 4.1. From the definition of $j^{a, b}$ (see 2.5) one easily derives $[X, Y]=\sum_{\ell=1}^{a} \operatorname{Im}\left(\bar{X}_{\ell} Y_{\ell}\right)+\sum_{\ell=a+1}^{a+b} \operatorname{Im}\left(Y_{\ell} \bar{X}_{\ell}\right)$, for $X=\left(X_{1}, \ldots, X_{a+b}\right), Y=\left(Y_{1}, \ldots, Y_{a+b}\right) \in$ $\mathfrak{v}=\mathbb{H}^{a+b}$, where $\operatorname{Im} q$ denotes the imaginary part of $q \in \mathbb{H}$, and $\bar{q}$ denotes the quaternionic conjugate of $q$. Let $x:=(1 / \sqrt{2}, 0, \ldots, 0,1 / \sqrt{2}), Y^{\mathfrak{v}}:=(j, 0, \ldots, 0, k), Y^{\mathfrak{z}}:=i$. Then $\left[Y^{\mathfrak{v}}, x\right]=$ $(-j-k) / \sqrt{2} \in \mathfrak{z}, j_{Y^{\mathfrak{z}}} Y^{\mathfrak{v}}=(j i, 0, \ldots, 0, i k)=(-k, 0, \ldots, 0,-j) \in \mathfrak{v}$, and $\left[j_{Y^{\mathfrak{j}}} Y^{\mathfrak{v}}, x\right]=(k+$ 
$j) / \sqrt{2} \in \mathfrak{z}$. Hence, for $p:=\exp (x, 0) \in \tilde{N}^{a, b}$ and $Y:=L_{p *}\left(Y^{\mathfrak{v}}+Y^{\mathfrak{z}}\right)$ we have by Lemma 4.3(ii): $\left(\nabla_{Y} \operatorname{ric}\right)(Y, Y)=\langle-j-k, k+j\rangle / 2=-1 \neq 0$.

Theorem 4.4 and Theorem 3.1 now imply:

Corollary 4.5. There exist pairs of compact isospectral Riemannian manifolds (here: $N^{a+b, 0}$ and $N^{a, b}$ with $\left.a, b>0\right)$ in which the first manifold is weakly locally symmetric while the second is not of type $\mathcal{A}$.

Recall from the Introduction that this corollary proves our Main Theorem 1.3.

\section{REFERENCES}

[1] T. Arias-Marco, Study of homogeneous D'Atri spaces of the Jacobi operator on g.o. spaces and the locally homogeneous connections on 2-dimensional manifolds with the help of MATHEMATICA ${ }^{\circledR}$. Dissertation, Universitat de València, Valencia, Spain (2007) ISBN: 978-84-370-6838-1, http://www.tdx.cat/TDX-0911108110640.

[2] T. Arias-Marco, The classification of 4-dimensional homogeneous D'Atri spaces revisited, Differential Geom. Appl. 25 (2007), 29-34.

[3] T. Arias-Marco, O. Kowalski, The classification of 4-dimensional homogeneous D'Atri spaces, Czechoslovak Math. J. 58(133) (2008), 203-239.

[4] J. Berndt, F. Prüfer, L. Vanhecke, Symmetric-like Riemannian manifolds and geodesic symmetries, Proc. Roy. Soc. Edinburgh Sect. A 125 (1995), no. 2, 265-282.

[5] J. Berndt, F. Tricerri, L. Vanhecke, Generalized Heisenberg groups and Damek-Ricci harmonic spaces, Lecture Notes in Mathematics 1598, Springer-Verlag, Berlin/Heidelberg/New York, 1995.

[6] J. Berndt, L. Vanhecke, Two natural generalizations of locally symmetric spaces, Differential Geom. Appl. 2 (1992), 57-80.

[7] J. Berndt, L. Vanhecke, Geometry of weakly symmetric spaces, J. Math. Soc. Japan 48 (1996), 745-760.

[8] J.T. Cho, K. Sekiwaga, L. Vanhecke, Volume-preserving geodesic symmetries on four-dimensional Hermitian Einstein spaces, Nagoya Math. J. 146 (1997), 13-29.

[9] J.E. D’Atri, H.K. Nickerson, Divergence preserving geodesic symmetries, J. Diff. Geom. 3 (1969), 467-476.

[10] P. Eberlein, Geometry of two-step nilpotent groups with a left invariant metric, Ann. Sci. Ecole Norm. Sup. (4) 27 (1994), 611-660.

[11] C.S. Gordon, R. Gornet, D. Schueth, D. Webb, E.N. Wilson, Isospectral deformations of closed Riemannian manifolds with different scalar curvature, Ann. Inst. Fourier 48 (1998), no. 2, 593-607.

[12] A. Gray, Einstein-like manifolds which are not Einstein, Geometriae Dedicata 7 (1978), 259-280.

[13] S. Kobayashi, K. Nomizu, Foundations of Differential Geometry. Vol. I, Interscience, New York/London, 1963.

[14] O. Kowalski, Some curvature identities for commutative spaces, Czechoslovak Math. J. 32 (1982), 389-396.

[15] O. Kowalski, Spaces with volume-preserving symmetries and related classes of Riemannian manifolds, Rend. Sem. Mat. Univ. Politec. Torino, Fascicolo Speciale (1983), 131-158.

[16] O. Kowalski, F. Prüfer, On probabilistic commutative spaces, Monasth. Math. 107 (1989), 57-68.

[17] O. Kowalski, F. Prüfer, L. Vanhecke, D'Atri spaces, Prog. Nonlinear Differential Equations Appl. 20 (1996), $241-284$.

[18] J. Lauret, Commutative spaces which are not weakly symmetric, Bull. London Math. Soc. 30 (1998), 29-36.

[19] H. Pedersen, P. Tod, The Ledger curvature conditions and D'Atri geometry, Differential Geometry and its Applications 11 (1999), 155-162.

[20] P.H. Roberts, H.D. Ursell, Random walk on a sphere and on a Riemannian manifold, Philos. Trans. Roy. Soc. London, Ser. A, 252 (1960), 317-356.

[21] D. Schueth, Isospectral manifolds with different local geometries, J. reine angew. Math. 534 (2001), 41-94.

[22] K. Sekiwaga, L. Vanhecke, Volume-preserving geodesic symmetries on four-dimensional 2-stein spaces, Kodai Math. J. 9 (1986), 215-224.

[23] K. Sekiwaga, L. Vanhecke, Volume-preserving geodesic symmetries on four-dimensional Kähler manifolds, Lecture Notes in Math. 1209 (1986), 275-291.

[24] A. Selberg, Harmonic analysis and discontinuous groups in weakly symmetric Riemannian spaces with applications to Dirichlet series, J. Indian Math. Soc. 20 (1956), 47-87. 
[25] Z.I. Szabó, Spectral theory for operator families on Riemannian manifolds, Proc. Symp. Pure Math. 54, 3 (1993), 615-665.

[26] Z.I. Szabó, Locally non-isometric yet super isospectral spaces, Geom. Funct. Anal. 9 (1999), no. 1, $185-214$.

[27] L. Vanhecke, T.J. Willmore, Interaction of tubes and spheres, Math. Ann. 263 (1983), 31-42.

Departamento de Matemáticas, Universidad de Extremadura, 06071 Badajoz, Spain

E-mail address: ariasmarco@unex.es

Institut für Mathematik, Humboldt-Universität Zu Berlin, D-10099 Berlin, Germany

E-mail address: schueth@math.hu-berlin.de 